\title{
Growth, Neurobehavioral and Circadian Rhythm Development in Newborn Baboons
}

\author{
MAJID MIRMIRAN, LUCA BERNARDO, SUSAN L. JENKINS, XIAO HONG MA, \\ J. THOMAS BRENNA, AND PETER W. NATHANIELSZ
}

\begin{abstract}
Laboratory for Pregnancy and Newborn Research, Department of Biomedical Sciences, T9 020 Veterinary Research Tower, College of Veterinary Medicine, Cornell University, Ithaca, New York 14853, U.S.A. [S.L.J., X.H.M., P.W.N.]; Department of Pediatrics, 8 Via A. di Rudini, University of Milan, San Paolo Hospital, 20142 Milan, ITALY [L.B.]; Netherlands Institute for Brain Research, Meibergdreef 33, 1105 AZ Amsterdam Zuidoost, The Netherlands [M.M.]; and Nutritional Sciences, Savage Hall, Cornell University, Ithaca, New York 14853, U.S.A. [J.T.B.]
\end{abstract}

\begin{abstract}
ABST
We measured body temperature continuously using telemetry
to determine the development of circadian rhythmicity in neo-
natal baboons after birth. Twelve fetal baboons (nine males and
three females) of known gestational age ranging from 167 to
$193 \mathrm{~d}$ were studied. We eliminated the influence of maternal
factors by hand rearing these infants from the moment of birth
until 45 d of life. All infants showed steady growth in body
weight, head circumference, and crown-rump length. Neurobe-
havioral responses including visual and auditory orientation,
motor maturity, irritability, and consolability increased as a
\end{abstract}
function of age. Circadian rhythms of body temperature were present in the second week of life, and the amplitude of this rhythm increased throughout the developmental period studied. The increase in the amplitude of circadian body temperature rhythm independent of environmental time cues may indicate the maturation of the brain. These neonatal nonhuman primates offer an excellent model for studying neurobehavioral development and maturation of circadian rhythms while controlling external factors in a manner that is not possible with human neonates.

(Pediatr Res 49: 673-677, 2001)
Circadian rhythms with a periodicity of approximately $24 \mathrm{~h}$ are endogenous rhythms that regulate many physiologic functions. These rhythms are generated by a biologic clock located in the suprachiasmatic nucleus in the brain (1). Appropriate appearance of perinatal circadian rhythms, both fetal and neonatal, is a fundamental expression of normal brain maturation (2). The extent to which these rhythms are present at birth and/or mature during the early neonatal period has been studied extensively in rodents (3). However, there are very few relevant studies on maturation in primates, either human or nonhuman $(4,5)$. The few recent nonhuman primate neonatal studies that exist relate only to the responsiveness of these rhythms to light $(6,7)$.

Maternal endocrine and other rhythms, however, have been investigated in nonhuman primates. Some have been shown to be truly circadian, e.g. body temperature and uterine myometrial activity $(8,9)$. Maternal hormonal rhythms have also been

Received July 13, 2000; accepted October 12, 2000.

Correspondence: Peter W. Nathanielsz, M.D., Ph.D., Sc.D., James Law Professor of Reproductive Physiology, Director, Laboratory for Pregnancy and Newborn Research, Box 16, T9 020 Veterinary Research Tower, College of Veterinary Medicine, Cornell University, Ithaca, NY 14853, U.S.A.; e-mail: pwn1@cornell.edu

Supported by NIH grant NS34805.

Presented in the APS-SPR meeting, May 1999, San Francisco, California, and published in abstract form in Pediatric Research 45:56A. shown to persist in the absence of external cues, the ultimate criterion for a circadian rhythm (10). In addition, several other studies have demonstrated 24-h maternal rhythms in human and nonhuman primates $(11,12)$ during the last trimester of gestation. These maternal rhythms, too, may yet prove to be truly circadian; however, they have not yet been shown to free run in the absence of external cues.

The purpose of the present study was to determine whether neonatal baboons express a clear circadian rhythm in body temperature in the absence of postnatal environmental time cues including those received from their mothers. In addition, we wished to evaluate any increase in the amplitude of the rhythm as a function of postnatal age. Growth and neurobehavioral development were evaluated at weekly intervals.

\section{METHODS}

Twelve pregnant baboons (Papio papio) of known gestational age (term is approximately $180 \mathrm{~d}$ gestation) based on either exact breeding dates and/or prenatal ultrasound determination were studied. Baboons were maintained in individual cages and acclimated as previously described in detail $(8,10)$. All procedures were approved by the Cornell University Animal Care and Use Committee. All facilities were approved by the American Association for the Accreditation of Laboratory 
Animal Care. The baboons received food and water ad libitum and daily vitamins and sweets/fruit supplements. The environmental light-dark cycle was automatically regulated with $14 \mathrm{~h}$ of light and $10 \mathrm{~h}$ of dark (14L-10D). Repeated ultrasound evaluations were made throughout the pregnancy to confirm the date and control for possible intrauterine fetal growth retardation. Twelve normally developed fetuses (nine males and three females) with gestational age ranging from 167 to 193 d were studied. Eight fetuses were delivered in the early afternoon by cesarean section. Four fetuses delivered spontaneously at night and were removed from their mothers early the following morning. Infants were maintained under constant low lighting throughout the study.

Rearing and testing protocols were developed from procedures developed by Ruppenthal (13) and Schnieder and Suomi (14) for hand rearing and neurodevelopmental examination of neonatal rhesus monkeys. Upon delivery, a modified Apgar score was obtained (Table 1). Infants were then moved into the nursery room while still inside the incubator. For the first few days of life, they were maintained inside the incubator until they had a stable body temperature (approximately $96-99^{\circ} \mathrm{F}$ ) and heart rate $(>160)$ with no bradycardia when measured at least four times daily. Neonatal surgery was conducted at 2-5 d of age to place a telemeter to monitor core body temperature. After placement of the telemeter, each infant was moved to an individual cage in the same nursery room.

Feeding procedures. Commercial milk formula (ENFAMIL with iron) was freshly prepared every day and was warmed in a microwave oven just before feeding. The first five feedings after birth were ENFAMIL $10 \%$ every $2 \mathrm{~h}$. From the sixth feeding until d 4, neonates were fed ENFAMIL 50\% every 2-4 h. From d 4, feeding was continued with ENFAMIL 100\% every $4 \mathrm{~h}$. Neonates were held up at least twice a day to learn to self-feed from the bottle in the cage. From 1 wk of age, the infant usually consumed sufficient milk to permit a variable feeding schedule that involved four feeds daily. Feeding was performed at random times during the intervals 0600-0800, $1000-1200,1500-1700$, and $2000-2300 \mathrm{~h}$ for the rest of the study period. The infants were monitored continuously throughout the day via a video camera connected to a timelapse video system situated in the adjacent monitoring room.

Surgery for placement of the intraabdominal telemetry device. At 2 to $5 \mathrm{~d}$ of age, after the neonate's clinical condition was stable, an intraabdominal telemeter was placed in 11 neonates. One infant was not implanted due to scheduling issues during the assigned time window. Neonates were anesthetized with $1 \mathrm{mg}$ Valium and $10 \mathrm{mg} / \mathrm{kg}$ ketamine. A radio transmitter (model TA11CTA-F40 or TIOETA-F20, Data Sciences International, St. Paul, MN, U.S.A.) approximately 3.0

Table 1. Modified Apgar score used for infant baboons

\begin{tabular}{lccc}
\hline \multicolumn{1}{c}{ Measure } & 0 & 1 & 2 \\
\hline Heart rate (beats/min) & - & $1-150$ & $>150$ \\
Respiration (breaths/min) & - & $<60$ & $>60$ \\
Muscle tone & - & some & flexed \\
Activity & - & passive & active \\
Color & gray & some gray & pink \\
Rectal temperature $\left({ }^{\circ} \mathrm{F}\right)$ & $<80$ & $80-90$ & $>90$ \\
\hline
\end{tabular}

$\times 0.75 \times 1.5 \mathrm{~cm}$ capable of recording core body temperature continuously for months was implanted in the left side of the peritoneal cavity and fixed to the anterior abdominal wall. After the operation, the infants were placed in their home cages $(18 \times 12 \times 14$ in $)$ and observed directly and monitored continuously in the nursery until they were fully recovered from the surgery. They resumed their normal levels of activity including self-feeding within $6 \mathrm{~h}$ after surgery. Cages were situated a half meter apart so that neonates could see and communicate with each other.

Telemetry receivers (model RLA2000, Data Sciences International, St. Paul, MN, U.S.A.) were mounted on either side of the cage to obtain signals from each freely moving infant. The signals were transmitted to the monitoring room next to the nursery room. A software program written in Lab View (National Instruments, Austin, TX, U.S.A.) continuously recorded infant body temperature. The data were also displayed on the computer monitor over each 24-h period. Body temperature signals were sampled at $1 \mathrm{~Hz}$. The telemetry system and closed time-lapse video system were designed to monitor four infants simultaneously throughout the entire study period. All body temperature data were stored on a personal computer for later analysis. We maintained the nursery room illumination at 25 lux continuously during the 24-h day throughout the study.

Anthropometric measures. The body weight of each neonate was measured every day before the morning feeding. Head circumference, crown-rump, and length of left foot were also measured weekly (13). Each anthropometry measure was repeated three times, and the mean of these values was used for final analysis.

Neurobehavioral examination. We have used modified neurobehavioral tests developed by Schneider and Suomi (14) for newborn rhesus monkeys. This battery of tests was modified by them from the Brazelton's Infant Behavioral Assessment Scale, which was originally developed for human infants (15). One investigator from our group (M.M.) visited the laboratory of Dr. M.L. Schneider (University of Wisconsin, Madison, WI, U.S.A.) to learn how to apply these tests to our newborn baboons. We measured four clusters of tests: visual response, auditory response, motor maturity, and state control. In short, for visual response, we measured visual orientation, duration of attention, distractibility, and visual follow of each infant of an inanimate object. We evaluated auditory orientation to the stimulus of a soft-sounding toy presented to the left and right ear. Measures of motor maturity included head control in supine and prone positions, tone, righting/balance, response speed, coordination, and amount/quality of spontaneous movements. State control was examined by measuring the level of irritability, consolability, and struggle/resistance of the baby during the entire neurobehavioral test. All measures were made every week and scored according to a scale developed by Schneider and Suomi (14): 0 (poor), 1 (intermediate), and 2 (fully developed).

Data analysis. Body temperature data averaged every $4 \mathrm{~s}$ from each infant were imported as text files into EXCEL (Microsoft). They were visually examined and edited for artifactual values above $40^{\circ} \mathrm{C}$ or below $35^{\circ} \mathrm{C}$, which occasionally occur due to failure of the telemetry receiver for $4 \mathrm{~s}$ due to 
alterations imposed by the animal's spontaneous movements. Moving-window averaging was performed using a 6-min window. Every week of data was analyzed separately. The maximum (peak) and minimum (trough) of the rhythm was defined for each given day. The amplitude of the body temperature rhythm was defined as the difference in degrees centigrade between the peak and trough and was later averaged for the number of days for each week. Data are provided throughout as mean \pm SEM. Two-way ANOVA and Student-NewmanKeul's post hoc test were used for statistical analysis of chronological age, sex, and sex by age interaction.

\section{RESULTS}

Neonatal condition at delivery. The data from birth records of all infants are presented in Table 2.

Neonatal growth profile. Growth was significantly $(p<$ 0.001 ) increased as a function of age for all parameters measured, independent of sex (Fig. 1). No sex by age interaction was found.

Neurobehavioral maturation. Neurobehavioral maturity measured by the modification of the Schneider and Suomi (14) test for the four major clusters of tests (Fig. 2) showed a significant age effect $(p<0.001)$. For state control, a significant $(p<0.05)$ sex by age interaction was present.

Circadian rhythms in neonatal body temperature. An example of five weekly continuous telemetry monitoring of neonatal body temperature of one subject is shown in Figure 3. This representative plot shows the reliability of this procedure for continuous monitoring without the need for frequent handling of the neonate. The development of the mean amplitude of the body temperature rhythm of 11 neonatal baboons is shown in Figure 4. A clear amplitude of $>1{ }^{\circ} \mathrm{C}$ in body temperature was found during the second week of life in all neonates. The amplitude of this rhythm showed significant age effect $(p<0.02)$. No significant effect of gestational age, sex, or sex by age interaction was found. During the fifth week of life, the amplitude of body temperature rhythm was significantly increased compared with the second and third weeks.

Because the telemeters were implanted over the period 2 to $5 \mathrm{~d}$ of age, data were obtained from some newborns for $5 \mathrm{wk}$ (as the animal shown in Fig. 3) and in others for only 4 wk.

Table 2. Birth records of all infants

\begin{tabular}{|c|c|c|c|c|}
\hline Infant & $\mathrm{dGA}$ & Sex & $\begin{array}{c}\text { Birth wt } \\
(\mathrm{g})\end{array}$ & Apgar \\
\hline $1 \mathrm{M}$ & 167 & $\mathrm{M}$ & 710 & 9.5 \\
\hline $2 \mathrm{M}$ & 170 & $\mathrm{M}$ & 1176 & 12 \\
\hline $3 \mathrm{M}$ & 193 & $\mathrm{M}$ & 1215 & 12 \\
\hline $4 \mathrm{M}$ & 174 & $\mathrm{M}$ & 870 & 12 \\
\hline $5 \mathrm{M}$ & 175 & $\mathrm{M}$ & 908 & $*$ \\
\hline $6 \mathrm{M}$ & 178 & $\mathrm{M}$ & 716 & 9 \\
\hline $7 \mathrm{M}$ & 176 & $\mathrm{M}$ & 816 & $*$ \\
\hline $8 \mathrm{M}$ & 184 & $\mathrm{M}$ & 1102 & 7.5 \\
\hline $9 \mathrm{M}$ & 191 & $\mathrm{M}$ & 980 & 8 \\
\hline $1 \mathrm{~F}$ & 189 & $\mathrm{~F}$ & 839 & 12 \\
\hline $2 \mathrm{~F}$ & 180 & $\mathrm{~F}$ & 751 & 9 \\
\hline $3 \mathrm{~F}$ & 187 & $\mathrm{~F}$ & 849 & $*$ \\
\hline
\end{tabular}

* Delivery was unattended; no Apgar score available.

dGA indicates days gestational age.
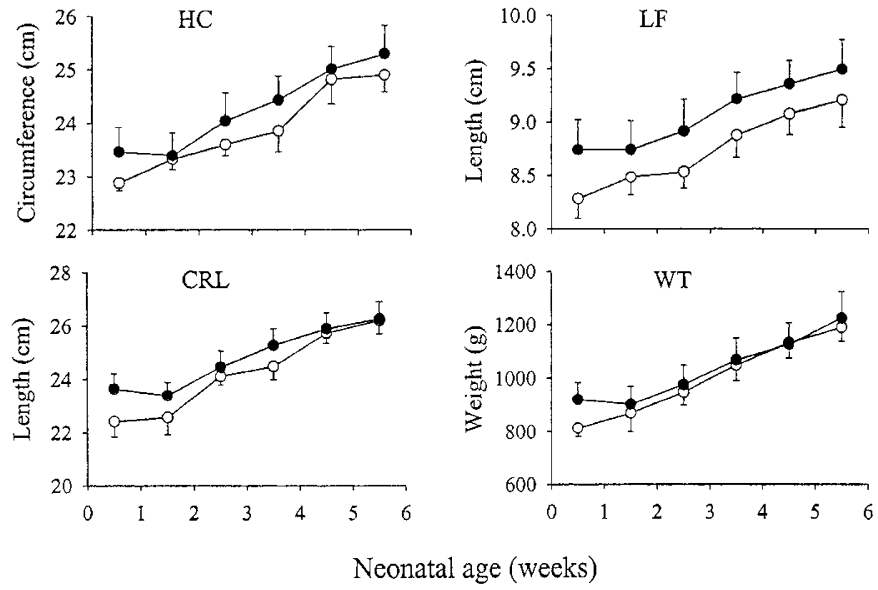

Figure 1. Weekly anthropometric measures showing the development of head circumference $(H C)$, left foot $(L F)$, crown-rump length $(C R L)$, and body weight $(W T)$ in female $(O ; n=3$, mean \pm SEM $)$ and male $(\bullet ; n=9$, mean \pm SEM) hand-reared newborn baboons.
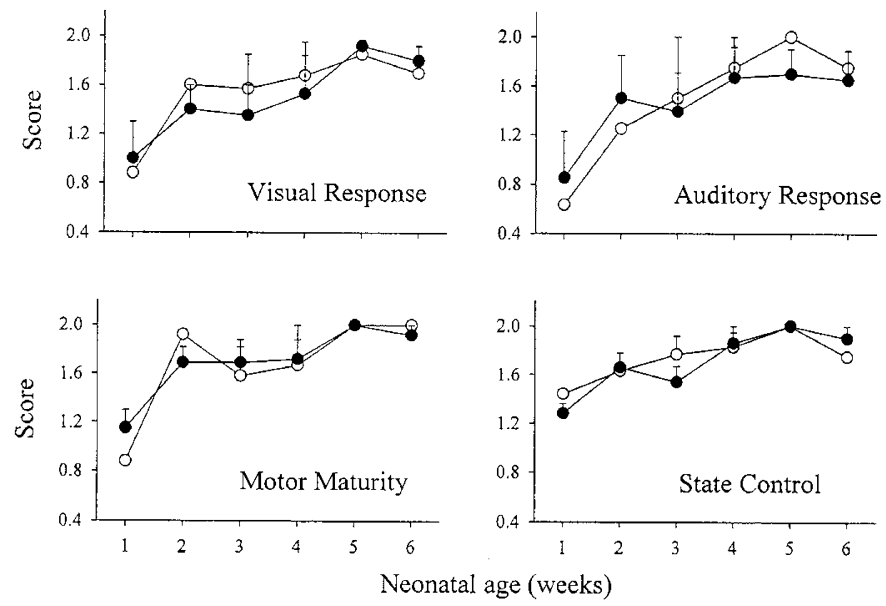

Figure 2. Development of visual response, auditory response, motor maturity, and state control in female $(O ; n=3$, mean \pm SEM $)$ and male $(-; n=9$, mean \pm SEM) hand-reared newborn baboons. Absence of error bar indicates $\mathrm{SEM}=0$.

Weekly data of all infants were pooled and expressed as $1-2$, $2-3,3-4$, and $4-5$ wk of age in Figure 4.

\section{DISCUSSION}

To prove definitively that rhythms in the newborn are truly circadian, it is necessary to hand rear the infants to exclude maternal influences that may entrain the infant rhythm. We, therefore, adapted the procedures for hand rearing originally developed by Ruppenthal (13) for rhesus monkeys to the newborn baboon. To confirm the satisfactory nature of the rearing conditions, we evaluated several parameters of neonatal growth. Our data clearly show adequate neonatal development as indicated by growth patterns and neurobehavioral maturation. Although it is difficult to compare the developmental curves between two species, our findings on neonatal baboon growth are similar to those of Ruppenthal (13) in respect to body weight, head circumference, crown-rump, and left foot length in neonatal rhesus monkeys. Our neurobehavioral findings are also in agreement with the data from Schnei- 


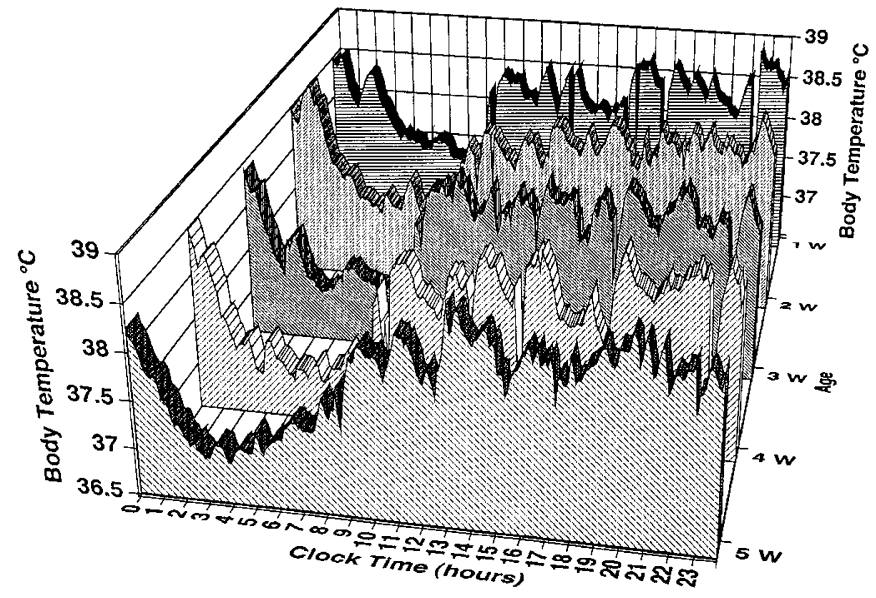

Figure 3. Development of body temperature circadian rhythm in an individual hand-reared newborn baboon at weekly intervals over the first $5 \mathrm{wk}$ of neonatal life.

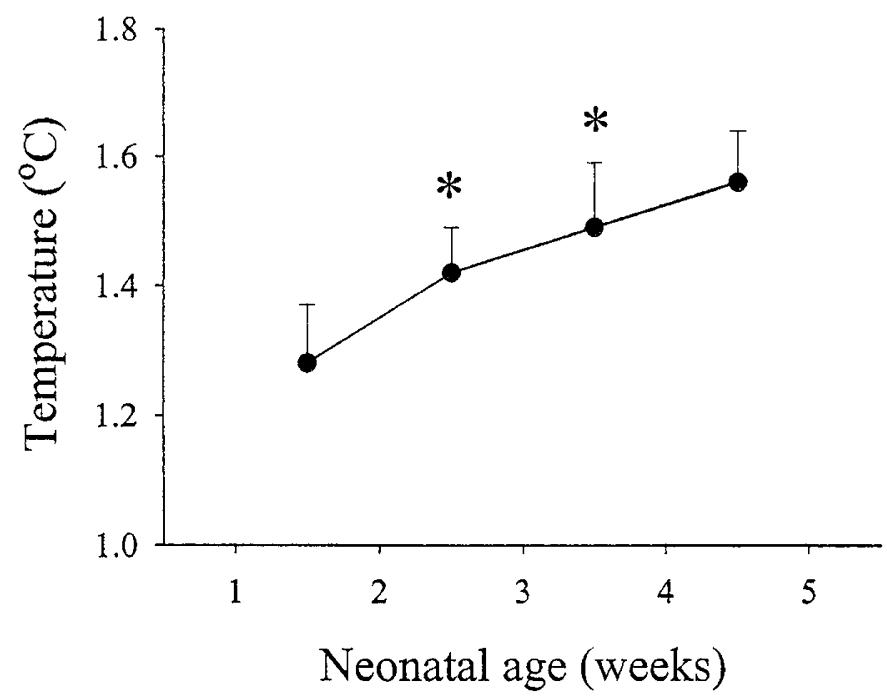

Figure 4. Development of the amplitude (mean \pm SEM) of the circadian rhythm in body temperature in 11 hand-reared newborn baboons over wk $2-5$ of neonatal life. ${ }^{*} p<0.05 v s$ wk 5 .

der and Suomi (14) in both maternally reared and hand-reared neonatal rhesus monkeys. Although hand rearing obviously removes postnatal maternal influences (including maternal milk) on growth and development of infants, hand rearing has the advantage of permitting frequent testing of the infants for maturation of brain and behavior without imposition of the repeated stress of maternal separation. It is for this reason that we separated the neonates from their mothers and hand reared them under uniform conditions in a specially designed nursery. Infants rapidly acclimated to the investigators and were very easy to handle throughout the experimental period.

A significant circadian rhythm in neonatal body temperature recorded by telemetry in an undisturbed constant environment was found from the second week of age. This conclusion is restricted to the amplitude of body temperature rhythm within the 6-wk study period. Because a period of time was needed to implant the telemetry sensor and acquire sufficient days of body temperature data, at present we cannot determine when exactly this rhythm appears or indeed whether it was present at birth. The amplitude of the body temperature rhythm significantly increased as a function of age. Time of onset, offset, and rate of rise and fall of body temperature as well as its phase response curve to light or other environmental time cues still need to be studied.

We have recently shown that the amplitude of the body temperature rhythm is similar in preterm and term human infants when corrected for age (16). At 1 mo of postnatal age, the amplitude of the body temperature rhythm in human neonates is approximately $0.7^{\circ} \mathrm{C}$, half that which we have demonstrated in the neonatal baboon at $1 \mathrm{mo}$ of age. At $3 \mathrm{mo}$ of age, the human neonate rhythm has an amplitude of approximately $1.1^{\circ} \mathrm{C}(5)$. It is interesting to note that in the latter study, a small but significant body temperature rhythm was present in preterm infants at 35-37 wk postconceptional age and more than $14 \mathrm{~d}$ postnatal age. However, preterm infants at 32-34 wk postconceptional age less than $14 \mathrm{~d}$ postnatal age showed only ultradian rhythms. Term gestational age for baboons in the colony studied is generally considered to be $175-185 \mathrm{~d}$ postconceptual age, but all nonhuman primates show a wide range of gestations at normal vaginal delivery. The gestational age of our infants varied from 167 to $193 \mathrm{~d}$, three less than 175, six in the normal window, and three greater than $185 \mathrm{~d}$ gestational age. We could not find any significant gestational age-dependent or prematurity effect. The limited number of animals per gestational age group and only three that were marginally preterm make it very difficult to draw any firm conclusion regarding the effects of gestational age on the development of circadian rhythms in this study. Due to the high risk of preterm labor in primates, it is not possible to implant the telemetry prenatally and study the infants right after birth. Future research similar to the study recently published (7) in which baboons with gestational age of $125 \mathrm{~d}$ were used is required to study the effect of prematurity and to establish how early these rhythms are developed.

The early appearance of a significant body temperature rhythm and its rapid development are also compatible with the view that the baboon neonate matures earlier and more rapidly than the human neonate. Our neurobehavioral results are also in support of earlier and more rapid maturation in baboons (13). In three animals under a continuous dim light condition, Rivkees et al. (6) demonstrated an appearance of circadian rest-activity rhythm as early as $2-3$ wk of postnatal age, very similar to our study. They cautioned that this rhythm may have been influenced by the light-dark cycle in which the dams were maintained before delivery. However, some of the data from the same investigators (6), as well as earlier elegant studies in rodents by Davis (17), are supportive of the notion that these rhythms are endogenous and are dependent on the integrity and maturation of the infant brain. A more recent interesting study that investigated the early development of the infant biologic clock (7) clearly showed light altered c-fos mRNA in the suprachiasmatic nucleus of the premature baboon as early as $125 \mathrm{~d}$ gestation. The authors further indicate the lack of previous data on humoral or behavioral outputs of the circadian system in neonatal baboons. Our study provides a clear indication that these rhythms are functional, at least for the regulation of body temperature, as early as the second week of 
postnatal life in animals delivered around term. More recent findings in humans, contrary to the earlier studies, are also in favor of an early maturation of circadian rhythms $(18,19)$. At present, there is no evidence of the functional significance of body temperature circadian rhythm in neonatal primates, including human newborns. One may argue that this rhythm may facilitate adaptation to environmental time cues, including maternal and environmental light-dark cycle, during the early neonatal period. We speculate that the nighttime body temperature trough in neonates helps to increase the length of nighttime sleep, thereby more rapidly synchronizing the monophasic sleep-wake pattern of the infant with its caregiver (19), a very beneficial adaptation for both mother and neonate.

Our study demonstrates a clear circadian rhythm in body temperature during early neonatal life. We agree with Hao and Rivkees $(7,20)$ that availability and study of a nonhuman primate animal model is crucial to define more precisely the nature, mechanisms, and maturation of human circadian rhythms in the perinatal period. A regular light-dark cycle in which human neonates are maintained in the neonatal intensive care units has been shown to accelerate sleep, growth, and clinical outcome $(21,22)$. Although all animals have circadian rhythms, primates including human are monophasic sleepers and differ from the commonly studied experimental species. Studies in rodents and other nonprimate models are of limited value in this situation. Rats are altricial mammals in which most of the period of rapid brain development takes place postnatally. Sheep, on the other hand, are more precocial than human infants, with most if not all of their brain structures developing prenatally, and have adult-like polyphasic sleep patterns at birth. The time course of prenatal and postnatal development of nonhuman primates makes them the most suitable animal models for human brain development.

\section{REFERENCES}

1. Moore RY 1997 Circadian rhythms: basic neurobiology and clinical applications. Ann Rev Med 48:253-266
2. Mirmiran M, Lunshof S 1996 Perinatal development of human circadian rhythms. Prog Brain Res 111:217-226

3. Reppert SM 1992 Perinatal development of a hypothalamic biological clock. Prog Brain Res 93:119-131

4. Mirmiran M, Kok JH 1991 Circadian rhythms in early human development. Early Hum Dev 262:121-128

5. Glotzbach SF, Edgar DM, Ariagno RL 1995 Biological rhythmicity in preterm infants prior to discharge from neonatal intensive care. Pediatrics 95:231-237

6. Rivkees SA, Hofman PL, Fortman J 1997 Newborn primate infants are entrained by low intensity lighting. Proc Natl Acad Sci USA 94:292-297

7. Hao HP, Rivkees SA 1999 The circadian clock of very premature primate infants is responsive to light. Proc Natl Acad Sci USA 96:2426-2429

8. Honnebier MBOM, Jenkins SL, Wentworth RA, Figueroa JP, Nathanielsz PW 1991 Temporal structuring of delivery in the absence of a photoperiod: preparturient myometrial activity of the rhesus monkey is related to maternal body temperature and depends on the maternal circadian system. Biol Reprod 45:617-625

9. Matsumoto T, Hess DL, Kaushal KM, Valenzuela GL, Yellon SM, Ducsay CA 1991 Circadian myometrial and endocrine rhythms in the pregnant rhesus macaque: effects of constant light and timed melatonin infusion. Am J Obstet Gynecol 165:1777-1784

10. Honnebier MBOM, Jenkins SL, Nathanielsz PW 1992 Circadian timekeeping during pregnancy: endogenous phase relationships between maternal plasma hormones and maternal body temperature rhythm in pregnant rhesus monkeys. Endocrinology 131:2052-2058

11. Lunshof S, Boer K, Wolf H, van Hoffen G, Bayram N, Mirmiran M 1998 Fetal and maternal diurnal rhythms during the third trimester of normal pregnancy: outcome of computerized analysis of continuous twenty-four hour fetal heart rate recordings. Am J Obstet Gynecol 178:247-254

12. Walsh SW, Ducsay CA, Novy MJ 1984 Circadian hormonal interactions among the mother, fetus, and amniotic fluid. Am J Obstet Gynecol 150:745-753

13. Ruppenthal GC (ed) 1979 Nursery Care of Non Human Primates, Plenum Press, NY, pp 1-350

14. Schneider ML, Suomi SJ 1992 Neurobehavioral assessment in rhesus monkey neonates (Macaca mulatta): developmental changes, behavioral stability, and early experience. Infant Beh Develop 15:155-177

15. Brazelton TB (ed) 1973 Neonatal Behavioral Assessment Scale (Clinics in Developmental Medicine No. 50). Heinemann, London, pp 1-66

16. Mirmiran M, Baldwin RB, Boeddiker M, Ariagno RL 1999 Development of circadian rhythms in premature infants. Pediatr Res 45:212A(abstr)

17. Davis DF 1991 Introduction. Suprachiasmatic Nucleus: The Mind's Clock. Klein DC, Moore RY, Reppert SM (eds) Oxford University Press, NY, pp. 375-390

18. Fukuda K, Ishihara K 1997 Development of human sleep and wakefulness rhythm during the first six months of life; discontinuous changes at the 7 th and 12th weeks after birth. Biol Rhythm Res 28:94-103

19. McGraw D, Hoffmann R, Harker C, Heman JH 1999 The development of circadian rhythms in a human infant. Sleep 22:303-310

20. Rivkees SA 1997 Developing circadian rhythmicity: basic and clinical aspects. Pediatr Clin North Am 44:467-487

21. Miller CL, White R, Whitman TL 1995 The effects of cycled versus noncycled lighting on growth and development in preterm infants. Infant Beh Dev 18:87-95

22. Mann NP, Haddow R, Stokes L, Goodley S, Rutter N 1986 Effect of night and day on preterm infants in a newborn nursery: randomized trial. Br Med J 293:12651267 